\title{
The spectrum of non-local discrete Schrödinger operators with a $\delta$-potential
}

\author{
Fumio Hiroshima ${ }^{1 *}$ and József Lőrinczi ${ }^{2}$
}

\begin{abstract}
The behaviour of the spectral edges (embedded eigenvalues and resonances) is discussed at the two ends of the continuous spectrum of non-local discrete Schrödinger operators with a $\delta$-potential. These operators arise by replacing the discrete Laplacian by a strictly increasing $C^{1}$-function of the discrete Laplacian. The dependence of the results on this function and the lattice dimension are explicitly derived. It is found that while in the case of the discrete Schrödinger operator these behaviours are the same no matter which end of the continuous spectrum is considered, an asymmetry occurs for the non-local cases. A classification with respect to the spectral edge behaviour is also offered.
\end{abstract}

Keywords: Discrete Schrödinger operator; Non-local Schrödinger operator; Modes and resonances; Rank-one perturbation; Bernstein function

\section{Introduction}

\subsection{Non-local discrete Schrödinger operators on lattice}

The spectrum of discrete Schrödinger operators has been widely studied for both combinatorial Laplacians and quantum graphs; for some recent summaries see $[3,4,7,9,11,16,19]$ and the references therein. Specifically, eigenvalue behaviours of discrete Schrödinger operators on $l^{2}\left(\mathbb{Z}^{d}\right)$ are discussed in e.g. $[2,8,10,12,13,15]$. However, for discrete non-local Schrödinger operators only few results are known. Typical examples include discrete fractional Schrödinger operators.

In this paper we define generalized discrete Schrödinger operators which include discrete fractional Schrödinger operators and others whose counterparts on $L^{2}\left(\mathbb{R}^{d}\right)$ are currently much studied $[5,6,17,18]$. In [14] we have introduced a class of generalized Schrödinger operators whose kinetic term is given by so called Bernstein functions of the Laplacian. These operators are non-local and via a Feynman-Kac representation generate subordinate Brownian motion killed at a rate given by the potential. Their discrete counterparts studied in this paper also have a probabilistic interpretation in that they generate continuous time random walks with jumps on $\mathbb{Z}^{d}$.

\footnotetext{
*Correspondence: hiroshima@math.kyushu-u.ac.jp

1 Faculty of Mathematics, Kyushu University, 744 Motooka, Nishiku, Fukuoka, Japan

Full list of author information is available at the end of the article
}

In the present paper we consider a class of Schrödinger operators obtained as a strictly increasing $C^{1}$-function of the discrete Laplacian and a $\delta$-potential. This includes, in particular, Bernstein functions (see below) of the discrete Laplacian. In the presence of a $\delta$-potential the above probabilistic picture then describes free motion with a "bump" which can be interpreted as an impurity in space. Our aim here is to investigate the spectrum of such operators, specifically, embedded eigenvalues and resonances at the edges of the continuous spectrum.

Let $d \geq 1$ and $L$ be the standard discrete Laplacian on $l^{2}\left(\mathbb{Z}^{d}\right)$ defined by

$$
L \psi(x)=-\frac{1}{2 d} \sum_{|x-y|=1}(\psi(y)-\psi(x)) .
$$

We give a remark on the definition of Laplacians for the reader's convenience. In the previous paper [15] we defined the discrete Laplacian by $L_{0} \psi(x)=\frac{1}{2 d} \sum_{|x-y|=1} \psi(y)$, and found that the spectrum of $L_{0}$ is the closed interval $[-1,1]$. In this paper we define the negative Laplacian by $\psi(x) \rightarrow$ $\frac{1}{2 d} \sum_{|x-y|=1}(\psi(y)-\psi(x))$, and flipping the signature, we define the positive Laplacian (1.1). Thus the spectrum of $L$ is positive, i.e., $\sigma(L)=[0,2]$. Hence we can consider the Bernstein functions of $L$. Also, let $V(x)=v \delta_{x, 0}$ be $\delta$ potential with mass $v$ concentrated on $x=0$, i.e., $V \psi(x)=$ 0 for $x \neq 0$ and $V \psi(x)=v \psi(x)$. Then the operator

$$
h=L+v \delta_{x, 0}, \quad v \in \mathbb{R}
$$

\section{是 Springer}


is the discrete Schrödinger operator with $\delta$-potential. In order to define a non-local version of $h$, we use Fourier transform on $l^{2}\left(\mathbb{Z}^{d}\right)$. Let $\mathbb{T}^{d}=[-\pi, \pi]^{d}$ be the $d$ dimensional torus, and set

$$
\mathscr{H}=L^{2}\left(\mathbb{T}^{d}\right)
$$

The scalar product on $\mathscr{H}$ is denoted by $(f, g)=$ $\int_{\mathbb{T}^{d}} \bar{f}(\theta) g(\theta) d \theta$. The Fourier transform $\mathscr{F}: l^{2}\left(\mathbb{Z}^{d}\right) \rightarrow$ $\mathscr{H}$ is then defined by $\mathscr{F} \psi(\theta)=\sum_{n \in \mathbb{Z}^{d}} \psi(n) e^{-i n \cdot \theta}$ for $\theta=\left(\theta_{1}, \ldots, \theta_{d}\right) \in \mathbb{T}^{d}$. Then the discrete Laplacian $L$ transforms as

$$
\mathscr{F} L \mathscr{F}^{-1}=L(\theta),
$$

where

$$
L(\theta)=\frac{1}{d} \sum_{j=1}^{d}\left(\cos \theta_{j}+1\right),
$$

i.e., the right hand side above is a multiplication operator on $\mathscr{H}$. In this paper we use a non-local discrete Laplacian $\Psi(L)$ defined for a suitable function $\Psi$ by applying Fourier transform.

Definition 1. For a given $\Psi \in C^{1}((0, \infty))$ such that $d \Psi(x) / d x>0, x \in(0, \infty)$, we define the non-local discrete Laplacian $\Psi(L)$ by

$$
\Psi(L)=\mathscr{F}^{-1} \Psi(L(\theta)) \mathscr{F} .
$$

Also, we call

$$
h=\Psi(L)+v \delta_{x, 0}, \quad v \in \mathbb{R},
$$

non-local discrete Schrödinger operator with $\delta$-potential.

An example of such a function is $\Psi(u)=u^{\alpha / 2}, 0<\alpha<$ 2 , which describes a discrete Laplacian of fractional order $\alpha / 2$. Other specific choices will be given in Example 1 below.

Under Fourier transform (1.7) is mapped into

$$
H_{v}=\mathscr{F} h \mathscr{F}^{-1}=\Psi(L(\theta))+v(\Omega, \cdot) \Omega,
$$

where $\Omega=(2 \pi)^{-d / 2} \mathbb{1} \in \mathscr{H}$.

Since $\sigma(L)=[0,2]$ and $\Psi$ is strictly increasing, it is immediate that $\sigma(\Psi(L))=\Psi([0,2])=[\Psi(0), \Psi(2)]$. In what follows we consider the spectrum of $H_{v}$ instead of $h$. Note that the map $\Phi \mapsto v(\Omega, \Phi) \Omega$ is a rank-one operator, and thus the continuous spectrum of the rank-one perturbation $H_{v}$ of $L$ is $[\Psi(0), \Psi(2)]$, for every $v \in \mathbb{R}$. See e.g. $[1,20]$ for rank-one perturbations.

\section{$1.2 \Psi(*)$-resonances and $\Psi(*)$-modes}

As it will be seen below, for a sufficiently large value of $-v>0$ there exists an eigenvalue $E_{-}(v)$ of $H_{v}$ strictly smaller than $\Psi(0)$. Suppose that $E_{-}(v) \uparrow \Psi(0)$ as $v \uparrow v_{0}$ with some $v_{0} \neq 0$. If $\Psi(0)$ is an eigenvalue of $H_{v_{0}}$, we call the eigenvector associated with $\Psi(0)$ a $\Psi(0)$-mode.
If $\Psi(0)$ is not an eigenvalue of $H_{v_{0}}$, we call it a $\Psi(0)$ resonance. Similarly, for a sufficiently large $v>0$ it will be seen that there exists an eigenvalue $E_{+}(v)$ strictly larger than $\Psi(2)$. Suppose that $E_{+}(v) \downarrow \Psi(2)$ as $v \downarrow v_{2}$ with some $v_{2} \neq 0$. If $\Psi(2)$ is an eigenvalue of $H_{v_{2}}$, we call the eigenvector associated with $\Psi(2)$ a $\Psi(2)$-mode, and a $\Psi$ (2)-resonance whenever $\Psi(2)$ is not an eigenvalue of $H_{v_{2}}$.

For the discrete Schrödinger operator $L+V$ these modes and resonances were studied in e.g. [15], in particular, their dependence on the dimension $d$. For $d=1,2$, there is no 0 -mode, 2-mode, 0 -resonance or 2-resonance, for $d=3,4$ there are 0 and 2-resonances, and for $d \geq 5$ there are 0 and 2 -modes. This shows that the eigenvalue behaviour at both edges ( 0 and 2 ) is the same. See Table 1.

As it will be seen below, for the case of the fractional Laplacian we have the remarkable fact that the edge behaviours are in general different at the two sides. See Table 2 and note that $\sigma(\sqrt{L})=[0, \sqrt{2}]$

\section{Eigenvalues}

\subsection{A criterion for determining the eigenvalues}

Consider the eigenvalue equation

$$
H_{\nu} \Phi=E \Phi
$$

or, equivalently,

$$
(E-\Psi(L(\theta))) \Phi=v(\Omega, \Phi) \Omega .
$$

We introduce the functions:

$$
\begin{aligned}
& I(x)=\int_{\mathbb{T}^{d}} \frac{d \theta}{|x-\Psi(L(\theta))|^{2}}, \\
& J(x)=\int_{\mathbb{T}^{d}} \frac{d \theta}{x-\Psi(L(\theta))} .
\end{aligned}
$$

The following result gives an integral test to spot the eigenvalues of $H_{\nu}$.

Lemma 1. $E$ is an eigenvalue of $H_{v}$ for a given $v$ if and only if $I(E)<\infty$ and $J(E) \neq 0$. Furthermore, if $E$ is an eigenvalue of $H_{v}$, then the coupling constant $v$ satisfies

$$
v=(2 \pi)^{d} / J(E) .
$$

Table 1 Modes and resonances of $L+V$

\begin{tabular}{lllll}
\hline & 2-mode & 2-res. & 0-mode & 0-res. \\
\hline$d=1$ & No & No & No & No \\
\hline$d=2$ & No & No & No & No \\
\hline$d=3$ & No & Yes & No & Yes \\
\hline$d=4$ & No & Yes & No & Yes \\
\hline$d \geq 5$ & Yes & No & Yes & No \\
\hline
\end{tabular}


Table 2 Modes and resonances of $\sqrt{L}+V$

\begin{tabular}{lllll}
\hline & $\sqrt{\mathbf{2}}$-mode & $\sqrt{\mathbf{2}}$-res. & 0-mode & 0-res. \\
\hline$d=1$ & No & No & No & No \\
\hline$d=2$ & No & No & No & Yes \\
\hline$d=3$ & No & Yes & Yes & No \\
\hline$d=4$ & No & Yes & Yes & No \\
\hline$d \geq 5$ & Yes & No & Yes & No \\
\hline
\end{tabular}

Proof. To show the necessity part, suppose that $E$ is an eigenvalue and $\Phi$ an associated eigenvector. Assuming $(\Omega, \Phi)=0$, we have $H_{\nu} \Phi=\Psi(L(\theta)) \Phi=E \Phi$. Since $\Psi(L(\theta))$ has no point spectrum, this is a contradiction. This gives $(\Omega, \Phi) \neq 0$ and $E-\Psi(L(\theta)) \neq 0$ for almost every $\theta \in \mathbb{T}^{d}$ with

$$
\Phi=\frac{(\Omega, \Phi)}{E-\Psi(L(\theta))} \in \mathscr{H} .
$$

Thus $I(E)<\infty$ follows, and $(\Omega, \Phi) \neq 0$ implies $J(E) \neq 0$.

For the sufficiency part, suppose now that $I(E)<\infty$ and $J(E) \neq 0$ hold. Define the $L^{2}\left(\mathbb{T}^{d}\right)$-function

$$
\Phi=\frac{c}{E-\Psi(L(\theta))}
$$

with a chosen $c$. It is straightforward to see that $\Phi$ satisfies $H_{v} \Phi=E \Phi$ whenever for $v$

$$
c\left(1-\frac{v}{(2 \pi)^{d}} J(E)\right)=0
$$

holds. By $J(E) \neq 0$ it follows that there exists $v$ such that (2.5) is satisfied, hence $E$ is an eigenvalue of $H_{v}$.

In order to investigate $\Psi(*)$-resonances and $\Psi(*)$ modes we use Lemma 1 and estimate the two integrals $I(E)$ and $J(E)$ at the two ends $E=\Psi(*)$ of the interval $[\Psi(0), \Psi(2)]$.

\subsection{The location of eigenvalues}

Lemma 2. Let $E \in \mathbb{R} \backslash[\Psi(0), \Psi(2)]$. Then there exists $v \neq 0$ such that $E$ is an eigenvalue of $H_{v}$.

Proof. In this case it is easily seen that $I(E)<\infty$ and $J(E) \neq 0$. Then $E$ is an eigenvalue and $v$ is given by (2.4).

Lemma 3. Let $\sigma_{p}\left(H_{v}\right)$ be the point spectrum of $H_{v}$. Then $\sigma_{p}\left(H_{v}\right) \cap(\Psi(0), \Psi(2))=\emptyset$, for every $v \neq 0$.

Proof. Due to monotonicity of $\Psi$, there is a unique $x \in$ $(0,2)$ such that $\Psi(E)=\Psi(x)$. Thus

$$
|E-\Psi(L(\theta))| \leq C\left|\frac{1}{d} \sum_{j=1}^{d}\left(\cos \theta_{j}+1-x\right)\right|
$$

with some $C>0$. Hence

$$
I(E) \geq \int_{\mathbb{T}^{d}} \frac{1}{\left|C \frac{1}{d} \sum_{j=1}^{d}\left(\cos \theta_{j}+1-x\right)\right|^{2}} d \theta .
$$

It is directly seen that the right hand side diverges, and thus the lemma follows.

Next consider the cases $E=\Psi(2)$ and $E=\Psi(0)$. For a systematic discussion of the eigenvalue behaviour of $H_{v}$ we introduce the following concept.

Definition 2. We say that $\Psi$ is of $(a, b)$-type whenever

$$
\begin{aligned}
& \lim _{x \rightarrow 0+} \frac{\Psi(x)-\Psi(0)}{x^{a}} \neq 0, \\
& \lim _{x \rightarrow 0} \frac{\Psi(2)-\Psi(2-x)}{x^{b}} \neq 0 .
\end{aligned}
$$

Lemma 4. Let $\Psi$ be of (a,b)-type. Then we have the following behaviour.

(1) $J(E) \neq 0$ for both $E=\Psi(0)$ and $E=\Psi(2)$.

(2) For $E=\Psi(2)$ we have that $I(E)<\infty$ if and only if $d \geq 1+4 a$, and $J(E)<\infty$ if and only if $d \geq 1+2 a$.

(3) For $E=\Psi(0)$ we have that $I(E)<\infty$ if and only if $d \geq 1+4 b$, and $J(E)<\infty$ if and only if $d \geq 1+2 b$.

Proof. Since $\Psi$ is strictly increasing, the first statement follows directly.

Let $\Psi$ be of $(a, b)$-type. Then we have at $\theta \approx(0, \ldots, 0)$,

$$
\Psi(2)-\Psi(L(\theta)) \approx\left(\frac{1}{2 d} \sum_{j=1}^{n} \theta_{j}^{2}\right)^{a}
$$

and at $\theta \approx(\pi, \ldots, \pi)$,

$$
\Psi(L(\theta))-\Psi(0) \approx\left(\frac{1}{2 d} \sum_{j=1}^{n}\left(\theta_{j}-\pi\right)^{2}\right)^{b} .
$$

Hence

$$
I(\Psi(2)) \approx \int_{\mathbb{T}^{d}} \frac{1}{\left(\sum_{j=1}^{n} \theta_{j}^{2}\right)^{2 a}} d \theta \approx \int_{0}^{1} \frac{r^{d-1}}{r^{4 a}} d r,
$$

and similarly

$$
J(\Psi(2)) \approx \int_{\mathbb{T}^{d}} \frac{1}{\left(\sum_{j=1}^{n} \theta_{j}^{2}\right)^{b}} d \theta \approx \int_{0}^{1} \frac{r^{d-1}}{r^{2 b}} d r .
$$

Thus the lemma follows for $E=\Psi(2)$. For the case of $E=\Psi(0)$ the proof is similar.

From these lemmas we can derive the spectral edge behaviour of $H_{v}$. The next theorem is the main result in this paper. 
Theorem 1. Assume that $\Psi$ is of $(a, b)$-type. Let

$$
v_{2}=(2 \pi)^{d} / J(\Psi(2))>0
$$

and

$$
v_{0}=(2 \pi)^{d} / J(\Psi(0))<0 .
$$

The spectral edge behaviour of $H_{\nu}$ is as follows.

(1) Suppose that $v>0$. Then the following cases occur:

(i) Let $d<1+2 b$. Then for all $v>0$ there exists an eigenvalue $E>\Psi(2)$.

(ii) Let $1+2 b \leq d<1+4 b$. Then for $v>v_{2}$ there exists an eigenvalue $E>\Psi(2)$, while for $v \leq v_{2}$ there is no eigenvalue.

(iii) Let $1+4 b \leq d$. Then for $v>v_{2}$ there exists an eigenvalue $E>\Psi(2)$, for $v=v_{2}$ the value $E=\Psi(2)$ is an eigenvalue, while $v<v_{2}$ there is no eigenvalue.

(2) Suppose that $v<0$. Then the following cases occur:

(i) Let $d<1+2 a$. Then for all $v<0$ there exists an eigenvalue $E<\Psi(0)$.

(ii) Let $1+2 a \leq d<1+4 a$. Then for $v<v_{0}$ there exists an eigenvalue $E<\Psi(0)$, while for $v \geq v_{0}$ there is no eigenvalue.

(iii) Let $1+4 a \leq d$. Then for $v<v_{0}$ there exists an eigenvalue $E<\Psi(0)$, for $v=v_{0}$ the value $E=\Psi(0)$ is an eigenvalue, while for $v>v_{0}$ there is no eigenvalue.

Proof. Consider the case $v>0$ and let $d<1+2 b$. Then for all $E>\Psi(2)$ we have $I(E)<\infty$ and $J(E) \neq 0$. Thus there exists $v$ such that $E$ is an eigenvalue of $H_{v}$.

Let $1+2 b \leq d<1+4 b$. Then for all $E>\Psi(2)$ we have that $I(E)<\infty$ and $J(E) \neq 0$. Thus $E$ is an eigenvalue of $H_{v}$. Since $J(E)<\infty$, it follows that $E \downarrow \Psi(2)$ as $v \downarrow v_{2}>0$. However, $E=\Psi(2)$ is not an eigenvalue since $I(E)=\infty$.

Let $d \geq 1+4 b$. Then for all $E>\Psi(2)$ we have $I(E)<\infty$ and $J(E) \neq 0$. Thus $E$ is an eigenvalue of $H_{v}$. Since $J(E)<$ $\infty$, we obtain $E \downarrow \Psi(2)$ as $v \downarrow v_{2}>0$. Since $I(E)<\infty$, we have that $E=\Psi(2)$ is also an eigenvalue. The cases for $v<0$ can be dealt with similarly.

Remark 1. Note that in general $-v_{0} \neq v_{2}$.

Remark 2. From the above it is seen that the spectral edge behaviour of $H_{v}$ depends on the dimension $d$ as well as on the parameters $a$ and $b$, and the result is different according to which edge is considered. For a summary see Table 3.

It is worthwhile to see the implications more closely for some specific choices of function $\Psi$.
Table $3 \Psi(0)$ and $\Psi(2)$-modes and resonances

\begin{tabular}{lll}
\hline $\boldsymbol{v}>\mathbf{0}$ & $\boldsymbol{\Psi}(\mathbf{2})$-mode & $\boldsymbol{\Psi}(\mathbf{2})$-res. \\
\hline$d<1+2 b$ & No & No \\
\hline $1+2 b \leq d<1+4 b$ & No & Yes \\
\hline $1+4 b \leq d$ & Yes & No \\
\hline $\boldsymbol{v}<\mathbf{0}$ & $\boldsymbol{\Psi}(\mathbf{0})$-mode & $\boldsymbol{\Psi}(\mathbf{0})$-res. \\
\hline$d<1+2 a$ & No & No \\
\hline $1+2 a \leq d<1+4 a$ & No & Yes \\
\hline $1+4 a \leq d$ & Yes & No \\
\hline
\end{tabular}

Example 1. (1) Discrete Schrödinger operator: Let $\Psi(u)=u$. Then $\Psi$ is of $(1,1)$-type and $H_{v}=L+V$. See Table 1.

(2) Fractional discrete Schrödinger operator: Let $\Psi(u)=u^{\alpha / 2}$ for $0<\alpha<2$. Then $\Psi$ is of $(\alpha / 2,1)$-type and $H_{v}=L^{\alpha / 2}+V$.

(3) Relativistic fractional discrete Schrödinger operator: Let $\Psi(u)=\left(u+m^{2 / \alpha}\right)^{\alpha / 2}-m$ for $0<\alpha<2$ and $m>0$. Then $\Psi$ is of $(1,1)$-type.

(4) Discrete jump-diffusion operator: Let $\Psi(u)=u+b u^{\alpha / 2}$ with $0<\alpha<2$. Then $\Psi$ is of $(\alpha / 2,1)$-type.

(5) Rotationally symmetric geometric discrete $\alpha$-stable operator: Let $\Psi(u)=\log \left(1+u^{\alpha / 2}\right)$ for $0<\alpha<2$. Then $\Psi$ is of $(\alpha / 2,1)$-type.

(6) Higher order discrete Laplacian: Let $\Psi(u)=u^{\beta}$ for $\beta>1$. Then $\Psi$ is of $(\beta, 1)$-type.

(7) Bernstein functions of the discrete Laplacian: Let $\Psi$ be a Bernstein function with vanishing right limits, i.e., $\Psi: \mathbb{R}^{+} \rightarrow \mathbb{R}^{+}$which can be represented in the form $\Psi(u)=b u+\int_{0}^{\infty}\left(1-e^{-u y}\right) v(d y)$, where $b \geq 0$ and $v$ is a Lévy measure with mass on $(0, \infty)$ satisfying $\int_{0}^{\infty}(1 \wedge y) v(d y)<\infty$. Then it follows that $\Psi^{\prime}(2)=b+\int_{0}^{\infty} y e^{-2 y} v(d y) \neq 0$. Furthermore, since $\Psi$ is concave, we have $a=\alpha / 2$ with some $\alpha \geq 2$. Hence $\Psi$ is of $(\alpha / 2,1)$-type with some $0 \leq \alpha \leq 2$. Note that the first five examples above are specific cases of Bernstein functions.

\section{A classification of spectral edge behaviour}

The functions $\Psi$ of the discrete Laplacian can be classified according to the behaviour of the eigenvalues at the two ends of the interval $[\Psi(0), \Psi(2)]$.

Definition 3. We call $\Psi$ normal type if $\Psi$ is $(1,1)$-type, and fractional type if $\Psi$ is $(\alpha / 2,1)$-type with $0<\alpha<2$.

The two types show qualitatively different behaviour and we discuss them separately. 


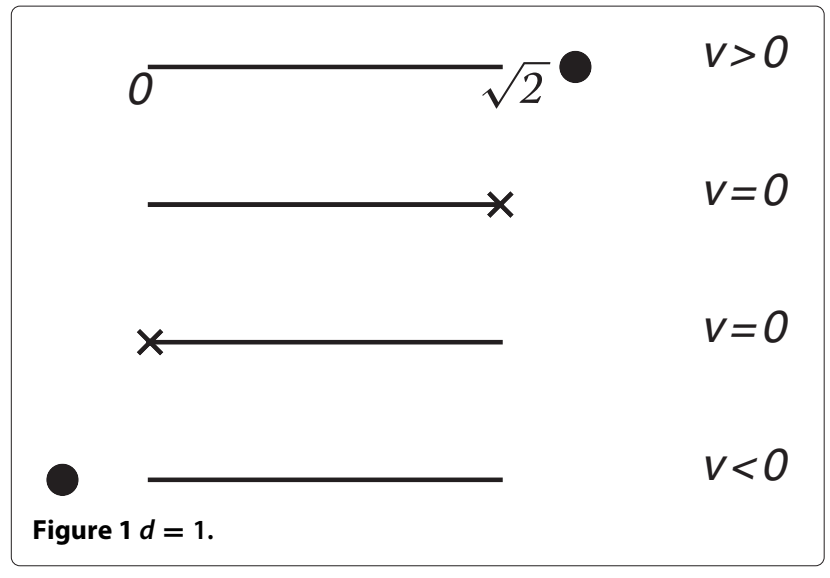

\subsection{Normal type}

Let $\Psi$ be of normal type. In this case the spectral edge behaviour is the same as that of the discrete Schrödinger operator $L+V$. The following result has been obtained in [15].

Proposition 1. Let $\Psi$ be normal type. We have the following cases.

(1) Let $d=1$ or 2 . For every $v>0$ there exists an eigenvalue $E>\Psi(2)$, and for every $v<0$ an eigenvalue $E<\Psi(0)$.

(2) Let $d=3$ or 4 . If $v>0$, then there exists $v_{2}>0$ such that for all $v>v_{2}$ an eigenvalue $E>\Psi(2)$ exists, and for $v \leq v_{2}$ no eigenvalue exists. If $v<0$, then there exists $v_{0}<0$ such that for all $v<v_{0}$ an eigenvalue $E<\Psi(0)$ exists, and for $v<v_{0}$ no eigenvalue exists.

(3) Let $d \geq 5$. If $v>0$, then there exists $v_{2}>0$ such that for all $v>v_{2}$ an eigenvalue $E>\Psi(2)$ exists, for $v=v_{2}$ the value $E=\Psi(2)$ is an eigenvalue, and for $v<v_{2}$ no eigenvalue exists. If $v<0$, then there exists $v_{0}<0$ such that for all $v<v_{0}$ an eigenvalue $E<\Psi(0)$ exists, for $v=v_{0}$ the value $E=\Psi(0)$ is an eigenvalue, and for $v>v_{0}$ no eigenvalue exists.

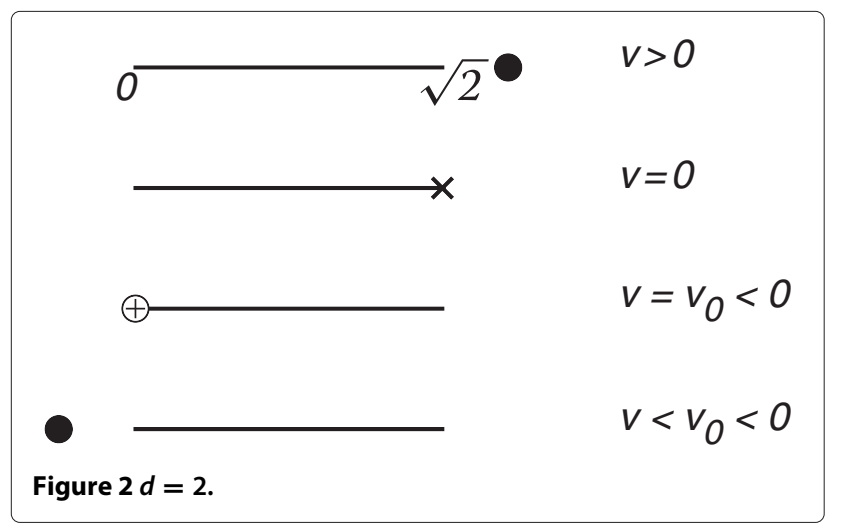

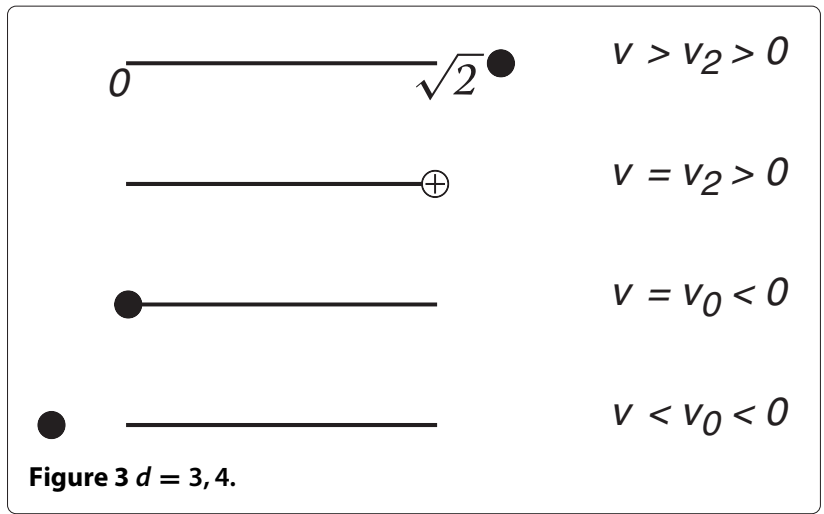

Thus the spectral edge behaviour for positive and negative $v$ is qualitatively the same, and the details only depend on the dimension $d$.

\subsection{Fractional type}

In the fractional type case we have the following spectral edge behaviour.

Theorem 2. Let $\Psi$ be of fractional type. The following cases occur.

(1) If $v>0$, then the spectral edge behaviour is the same as for normal type $\Psi$ with $v>0$.

(2) If $v<0$, then we have the following cases:

(i) Let $d<1+\alpha$. Then for every $v<0$ there exists an eigenvalue $E<0$.

(ii) Let $1+\alpha \leq d<1+2 \alpha$. There exists $v_{0}<0$ such that for all $v<v_{0}$ an eigenvalue $E<0$ exists, while for $v \leq v_{0}$ no eigenvalue exists.

(iii) Let $d \geq 1+2 \alpha$. There exists $v_{0}<0$ such that for all $v<v_{0}$ an eigenvalue $E<0$ exists, for $v=v_{0}$ the value $E=0$ is an eigenvalue, and for $v>v_{0}$ no eigenvalue exists.

In the fractional case it is seen that the edge behaviours for positive and negative $v$ are in general different

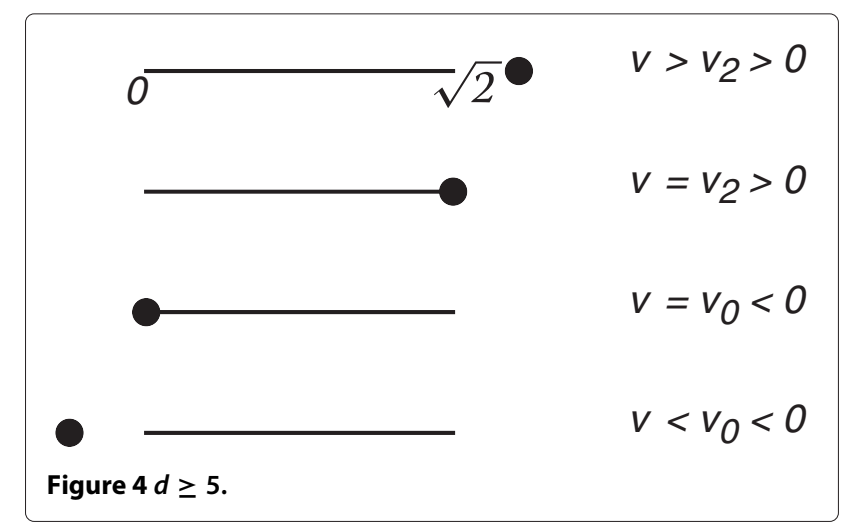


from each other, in contrast with the normal type case.

\subsubsection{The case of $\alpha=1$}

For $\alpha=1$ the spectral edge behaviour of $H_{v}=\sqrt{L}+V$ is displayed for dimensions $d=1, \ldots, 4$ and $d \geq 5$ in Table 2. We have displayed the specific situations in Figures 1, 2, 3 and 4 below, where $\oplus$ denotes a resonance, $\bullet$ an eigenvalue, and $\times$ denotes a value which is not an eigenvalue. For dimension $d=1,5$ the edge behaviours at 0 and $\sqrt{2}$ are symmetric. See Figures 1 and 4 . On the other hand for dimensions $d=2,3,4$ the edge behaviours at 0 and $\sqrt{2}$ are again different. See Figures 2 and 3.

\subsubsection{Massless and massive cases}

Consider the Bernstein function $\Psi(u)=\sqrt{u+m^{2}}-m$ with $m \geq 0$. This allows to define the relativistic discrete Schrödinger operator $\sqrt{L+m^{2}}-m+V$. Then it follows that $\Psi(u)$ is $(1,1)$-type for $m>0$, and $(1 / 2,1)$ type for $m=0$. In particular, the edge behaviours of $\sqrt{L+m^{2}}-m+V$ for $m>0$ are symmetric, and the edge behaviours of $\sqrt{L}+V$ and $\sqrt{L+m^{2}}-m+V$ are different. More generally, consider the Bernstein function $\Psi(u)=\left(u+m^{2 / \alpha}\right)^{\alpha / 2}-m$, with $0<\alpha<2$ and $m \geq$ 0 . This defines the rotationally symmetric relativistic $\alpha$ stable operator $\left(L+m^{2 / \alpha}\right)^{\alpha / 2}-m$. We conclude that $\Psi(u)$ is of (1,1)-type for $m>0$ but of $(\alpha / 2,1)$-type for $m=0$. Thus the edge behaviours of $\left(L+m^{2 / \alpha}\right)^{\alpha / 2}-m+V$ and $L^{\alpha / 2}+V$ are different.

\section{Acknowledgements}

FH is financially supported by Grant-in-Aid for Science Research (B) 23340032 from JSPS. JL thanks Institut Mittag-Leffler, Stockholm, for the opportunity to organise the research-in-peace workshop "Lieb-Thirring-type bounds for a class of Feller processes perturbed by a potential" during the period 25 July - 9 August 2013. JL also thanks London Mathematical Society for a travel grant to this workshop. FH thanks the invitation to this workshop, and we both express our gratitude to IML for the kind hospitality and inspiring work environment, where most of this paper has been prepared.

\section{Author details}

${ }^{1}$ Faculty of Mathematics, Kyushu University, 744 Motooka, Nishiku, Fukuoka, Japan. ${ }^{2}$ School of Mathematics, Loughborough University, Loughborough LE11 3TU, UK.

Received: 8 February 2014 Accepted: 3 March 2014

Published online: 04 October 2014

\section{References}

1. Aronszajn, N.: On a problem of Weyl in the theory of singular Strum-Liouville equations. Am. J. Math. 79, 597-610 (1957)

2. Bellissard, J., Schulz-Baldes, H.: Scattering theory for lattice operators in dimension $d \geq 3$. arXiv:1109.5459v2 (2012)

3. Berkolaiko, G., Carlson, R., Fulling, SA., Kuchment, PA. (Eds): Quantum graphs and their applications, Vol. 415 (2006)

4. Berkolaiko, G., Kuchment, PA.: Introduction to Quantum Graphs. AMS Math. Surv. Monographs 186 (2012)

5. Bogdan, K., Byczkowski, T.: Potential theory of Schrödinger operator based on fractional Laplacian. Probab. Math. Stat. 20, 293-335 (2000)

6. Bogdan, K., et al. (eds.): Potential analysis of stable processes and its extensions. Lecture Notes in Mathematics, Vol. 1980. Springer (2011)
7. Chung, F.: Spectral Graph Theory. CBMS Regional Conf. Series Math, Washington DC (1997)

8. Damanik, D., Killip, R., Simon, B.: Schrödinger operators with few bound states. Commun. Math. Phys. 258, 741-750 (2005)

9. Exner, P., Keating, JP., Kuchment, PA., Sunada, T., Teplyaev, A. (Eds): Analysis on graphs and its applications, Vol. 77 (2008)

10. Exner, P., Kuchment, PA., Winn, B.: On the location of spectral edges in $\mathbb{Z}$-peridoc media. J. Phys. A 43, 474022 (2010)

11. Grigor'yan, A.: Heat kernels on manifolds, graphs and fractals. In: European Congress of Mathematics, Barcelona, July 10-14, 2000, Progress in Mathematics 201, pp. 393-406. Birkhäuser, (2001)

12. Higuchi, Y., Matsumoto, T., Ogurisu, O.: On the spectrum of a discrete Laplacian on $\mathbb{Z}$ with finitely supported potential. Linear Multilinear Algebra 59, 917-927 (2011)

13. Higuchi, Y., Shirai, T.: The spectrum of magnetic Schrödinger operators on a graph with periodic structure. J. Funct. Anal. 169, 456-480 (1999)

14. Hiroshima, F., Ichinose, T., Lőrinczi, J.: Path integral representation for Schrödinger operators with Bernstein functions of the Laplacian. Rev. Math. Phys. 24, 1250013 (2012)

15. Hiroshima, F., Sasaki, I., Shirai, T., Suzuki, A.: Note on the spectrum of discrete Schrödinger operators. J. Math-for-Industry 4, 105-108 (2012)

16. Korotyaev, E., Saburova, N.: Schrödinger operators on periodic discrete graphs. arXiv:1307.1841 (2013)

17. Kulczycki, T.: Gradient estimates of $q$-harmonic functions of fractional Schrödinger operator. Potential Anal. 39, 69-98 (2013)

18. Lőrinczi, J., Małecki, J.: Spectral properties of the massless relativistic harmonic oscillator. J. Diff. Eq. 253, 2846-2871 (2012)

19. Post, O.: Spectral Analysis on Graph-Like Spaces, Lecture Notes in Mathematics, Vol. 2039. Springer (2012)

20. Simon, B., Wolff, T.: Singular continuous spectrum under rank one perturbations and localization for random Hamiltonians. Commun. Pure App. Math. 39, 75-90 (1986)

\section{doi:10.1186/s40736-014-0007-8}

Cite this article as: Hiroshima and Lőrinczi: The spectrum of non-local discrete Schrödinger operators with a $\delta$-potential. Pacific Journal of Mathematics for Industry 2014 6:7.

\section{Submit your manuscript to a SpringerOpen ${ }^{\circ}$ journal and benefit from:}

- Convenient online submission

- Rigorous peer review

- Immediate publication on acceptance

- Open access: articles freely available online

- High visibility within the field

- Retaining the copyright to your article

Submit your next manuscript at $>$ springeropen.com 